(Aus dem Institute für allgem. und exper. Pathologie der Universität Wien.

Vorstand: Hofrat Prof. Palta uf.)

\title{
Über \\ scheinbare Vaguslähmung (bei Muskarin, Physostigmin und anderen Giften sowie bei intrakardialer Drucksteigerung).
}

Von

Privatdozent Dr. C. J. Rothberser, Assistenten am Institute,
und Privatdozent Dr. H. Winterbers.

(Hierzu Tafel VI-IX.)

Den Ausgangspunkt der vorliegenden Untersuchungen bildet die merkwürdige Tatsache, dass bei Giften, denen eine spezifisch erregende Wirkung auf den Vagus zukommt, wie z. B. dem Muskarin, in einem gewissen Vergiftungsstadium die Hemmungsnerven bei noch nachweisbaren Erregungssymptomen dennoch gegenüber faradischer Reizung unerregbar scheinen.

Unsere Vermutung, dass es sich dabei nicht um eine wirkliche Lähmung der Vagi handeln, sondern. dass die Vaguswirkung nur durch das Auftreten automatischer Ventrikelkontraktionen verdeckt sein dürfte, erwies sich, wie im folgenden gezeigt werden soll, als richtig.

Es stellte sich weiter heraus, dass wir es hier mit einer überraschend weit verbreiteten Erscheinung zu tun haben, die nicht nur nach der Einwirkung von Giften, sondern auch unter dem Eiuflusse hoher intrakardialer Drucksteigerung nachzuweisen ist, und deren Kenntnis uns in den Stand setzt, eine Reihe strittiger physio- und pharmakologischer Fragen in befriedigender Weise zu lösen.

Unsere Versuche wurden ausnahmslos an Hunden ausgeführt. Zum Nachweis der Ventrikelautomatie bedienten wir uns der zu diesem Zwecke besonders geeigneten elektrographischen Methode. Dieselbe bringt gleichzeitig die chronotrope Komponente der Vagus- 
wirkung ausgezeichnet zur Anschauung, auf welche sich die in der Literatur vorliegenden, auf den Gegenstand unserer Untersuchung bezüglichen Angaben über Vaguslähmung beziehen.

Die Versuchstiere wurden mit Morphin und Curare vergiftet, der Thorax wurde geöffnet, das Herz blossgelegt. Zur Ableitung der Aktionsströme des Herzens verwendeten wir daumendicke in den Ösophagus und das Rektum eingeführte Neusilberelektroden. Die Vagi waren am Halse durchschnitten; ihre Erregbarkeit wurde fortlaufend kontrolliert. Sobald eine Anderung derselben eintrat, wurde sie durch eine elektrographische Aufnahme fixiert. Wir konnten so den Eintritt bzw. das Verschwinden der Vaguslähmung mit dem gleichzeitigen Verhalten des Elektrokardiogramms in Beziehung bringen.

In dieser Weise untersuchten wir den Einfluss von Muskarin, Physostigmin, Digitalis, Adrenalin und Strychnin sowie den der intrakardialen Drucksteigerung auf die Erregbarkeit des Vagus.

\section{Muskarin.}

In der Frage nach der Natur der Muskarinwirling spielt die von Petri ${ }^{1}$ ) entdeckte Tatsache, dass die Vagusreizung auf das muskarinisierte Froschherz nicht wirkt, eine interessante Rolle.

Petri's merkwürdiger. Befund wurde später in Laboratorium von v. Basch auch beim Hunde von Wei n-z weig ${ }^{2}$ ) erhoben, der fand, dass im Stadium der durch das Muskarin hervorgerufenen Verlangsamung selbst starke Reizung der Vagi nicht den geringsten Einfluss auf die Frequenz der Herzschläge bat.

"Würde aber", so schliesst W e in z w e ig aus seiner Beobachtung, „das Muskarin genau denselben Zustand erzeugen wie am normalen Herzen die Vagusreizung, so müsste es möglich sein, einen durch Muskarin verlangsamten Herzschlag durch Vagusreizung zum Stillstande zu bringen oder wenigstens noch mebr zu verlangsamen:"

Auch Berggrün ${ }^{3}$ ) sehliesst sich dieser Ansicht an und weist. zugleich die Annahme zurück, dass die Pulsverlangsamung bei der

1) Petri, Beitrag zur Lehre von den Hemmungsapparaten des Herzens. Dissert. Bern 1880.

2) Weinzweig, Über das Verhalten des mit Muskarin vergifteten Herzens gegen seine Nerven. Arch. f. Anat. u. Physiol. 1882 S. 527.

3) Berggrün, Experimenteller Beitrag zur Theorie der Muskarinwirkung. Zentralbl. f. Physiol. 1892, 27. Feb"., H. 24. 
Muskarinwirkung schon einem maximalen Erregungsgrade : des Vagusendapparates entspreche. Die durch Muskarin erzeugte Vagus. lähmung trete schon bei einer Art von Pulsverlangsamung auf, wie sie dem Effekte einer schwachen Vagusreizung am nicht vergifteten Herzen entspricht: "Wer mit Schmiedeberg", sagt dieser Autor weiter, „an der Meinung festhält, dass das Ausbleiben der Reaktion der Vagusreizung eine Lähmung des Endapparates der Vagi bedeute, muss also zu dem Schluss gelangen, dass das Muskarin, während es die Vagusendapparate im Herzen reizt und so zur Verlangsamung führt, dieselben zugleich lähmt und infolgedessen die Vagusreaktion vernichtet."

Im Widerspruche zu den eben genannten Autoren, ohne indessen ihre abweichenden Befunde zu erwähnen, behauptete Cushny ${ }^{1}$ ), dass der Vagus während der Muskarinwirkung solange seine normale Wirkung ausübt, als das Herz überhaupt schlägt.

Endlich liegt noch aus neuester Zeit eine Arbeit von Jonescu ${ }^{2}$ ) vor, der auf Anraten von Straub und offenbar in Unkenntnis der analogen Untersuchungen von Weinzweig und Berggrün das Problem, ob das Muskarin durch Vagusreizung wirkt, ebenfalls von der Überlegung aus prüfte, dass unter dieser Voraussetzung eine am Herzen nur submaximal sich äussernde Muskarinwirkung durch elektrische Vagusreizung verstärkt eventuell sogar maximal werden muss.

Seine Befunde stimmen mit jenen von Weinzweig und Berggrün in wesentlichen überein, indem auch Jonescu die submaximale Muskarinwirkung durch keinerlei Vagusreizung maximal machen, ja häufig vollständigen Verlust der Anspruchsfähigkeit der Hemmungsnerven feststellen konnte. Allerdings weist Jonescu bei Anwendung kleinster Muskarindosen bisweilen sogar als alleinige Muskarinwirkung eine gesteigerte Erregbarkeit der Vagi nach, die er jedoch nicht als den positiven Vorschlag der „im Prinzip lähmenden Muskarinwirkung" gelten lassen will.

Bei unseren an Hunden ausgeführten Experimenten fanden wir im Gegensatz zu Cushny und in Übereinstimmung mit den letzt-

1) Cushny, Über die Wirkung des Muskarins auf das Froschherz. Arch. f. exper. Pathol. u. Pharmakol. Bd. 31 S. 432.1893.

2) Jonescu, Über die Reizbarkeit der hemmenden Innervation des Froschherzens im Verlauf der Muskarinvergiftung. Arch. f. exper. Pathol. u Pharmakol. Bd. 60 S. 154. 1909. 
genannten Autoren sehr häufig, und zwar gewöhnlich unmittelbar nach dem Herzstillstande im Stadium der grössten Verlangsamung, aber auch bei schon wieder zunehmender Schlagfrequenz, die vor der Vergiftung gut wirksamen Vagi völlig unerregbar. Nach kürzerer oder längerer Zeit verschwand aber stets diese Unerregbarkeit, wobei besonders auffallend war, dass der Vagus ziemlich plötzlich wieder seine volle Wirkung entfaltete, eine Erscheinung, die auch in den Versuchsprotokollen von Joneseu hervortritt.

Da von der Schule Schmiedeberg's, wie in anderen Fällen, so auch hier namentlich gegen die Versuche von Petri der Einwand erhoben worden war, dass Verunreinigung durch eine von $\mathrm{Schmiedeberg}$ nachgewiesene atropinhaltige Base vorliege, so war es zunächst notwendig, den Einfluss dieser Fehlerquelle zu berücksichtigen. Man muss gewiss zugeben, dass unter der Voraussetzung einer raschen Inaktivierung bzw. Ausscheidung einer solchen atropinartigen Substanz die erwäbnte Erscheinung der an den Herzstillstand sich anschliessenden, relativ flüchtigen Vaguslähmung zwanglos zu erklären wäre, wenn die während der elektrischen Unerregbarkeit der Vagi bestehende Verlangsamung der Schlagfrequenz nicht als chronotrope Hemmungswirkung aufgefasst wird.

In der Tat wird auch der Herzstillstand bzw. die Verlangsamung der Schlagfolge von manchen Autoren, so insbesondere von Gaskell, als Folge eines direkten lähmenden Einflusses des Muskarins auf die Muskelsubstanz selbst oder auf die motorischen Herznervenapparate (Löwit) angesehen. Auch Weinzweig lehnt auf Grund de's Versagens der Vaguswirkung bei bestehender Verlangsamung die Schmiedeberg' sche Grundanschauung von der die Vagusapparate reizenden Wirkung des Muskarins ab und nimmt an, dass das Muskarin jene Vorrichtungen des Herzens, welche die Reize aufnehmen und ausgeben, derart verändert, dass die Herzkontraktion aufhört oder seltener wird. Hält man aber an der Hypothese von Schmiedeberg: fest und fübrt die Vaguslähmung auf eine Atropinwirkung zurück; : dann gerät man tatsächlich in das von Berggrün formulierte Dilemma, gleichzeitig eine Erregung und Lähmung der Vagusendapparate anzunehmen.

Gegen die im Laboratorium v. Basch's von Weinzweig und Berggrün ausgeführten Versuche kann jedoch der Einwand der Verunreinigung des gebrauchten Muskarinpräparates überhaupt nicht 
erhoben werden. Denn es wurde zu denselben ein genau nach der Schmiedeberg' schen Vorschrift durch v. Basch selbst im chemischen Institute von Ludwig in Wien hergestelltes Muskarin gebraucht.

$\mathrm{Zu}$ unseren Versuchen diente ein selbstbereiteter alkoholischer Auszug aus Fliegenpilzen, der in einer Menge von $0,1-0,2 \mathrm{ccm}$ bei Hunden von mittlerer Grösse $(12 \mathrm{~kg})$ langanhaltenden Herzstillstand hervorbrachte.

Wie schon eingangs erwähnt, gingen wir bei unseren Experimenten von dem Gedanken aus, dass der Vagus auf die in den Kanmern selbst entstehenden Kontraktionen kaum einen Einfluss hat, was namentlich für die chronotrope Komponente der Vaguswirkung gilt.

Von dieser Regel scheint es allerdings wenigstens beim Säugetierherzen Ausnahmen zu geben (Bayliss und Starling ${ }^{1}$ ), Hering ${ }^{2}$, $\mathrm{Rihl}^{3}$ ), doch ist unter allen Umständen die Vaguswirkung auf die selbsttätigen Kammern nach Erlanger ${ }^{4}$ ) sehr inkonstant und geringfügig.

Im allgemeinen wird man daher aus der Unerregbarkeit der Vagi bei automatisch schlagenden Kammern zunächst nur den Schluss ziehen dürfen, dass die Vaguswirkung durch die bestehende Kammerautomatie verschleiert wird.

Bei der Muskarinvergiftung lässt sich nun tatsächlich leicht nachweisen, dass das Stadium der scheinbaren Vagusparalyse stets genau zusammenfällt mit einer von den Vorhöfen unabhängigen Kammertätigkeit, und dass die Vagi in demselben Augenblicke wieder wirksam sind, in welchem die. Vorhöfe neuerdings die Führung des Herzschlages übernehmen.

In Fig. I a entfallen im Stadium der Verlangsamung bei Vergiftung

1) Bayliss und Starling, On some points in the innervation of the mammalian heart. Journ. of Physiol. vol. 13 p. 407. 1892.

2) Hering, Über die unmittelbare Wirkung des Accelerans und Vagus aut automatisch schlagende Abschnitte des Säugetierherzens. Pflüger's Arch. Bd. 108 S. 281 . 1905.

3) Rihl, Über Vaguswirkung auf die automatisch schlagenden Kammern des Säugetierherzens. Pflüger's Arch. Bd. 114 S. 545. 1906.

4) Erlanger, Über den Grad der Vaguswirkung auf die Kammern des Hundeherzens. Pflüger's Arch. Bd. 127 S. 77. 1909. 
mit Muskarin auf fünf Vorhof- $(P)$ nur drei Kammerschläge. Es besteht also zwischen $A$ und $V$ völlige Dissoziation. Bei der Inspektion war in diesem Falle, wie dies in diesem Stadium der Muskarinvergiftung gewöhnlich zutrifft, von einer Kontraktion der geblähten, scheinbar ganz untätigen Vorhöfe nicht das geringste wahrnehmbar. Eine Vagusreizung (R.-A. $50 \mathrm{~mm}$ unseres Induktoriums) hatte, wie dies in Fig. I b, der unmittelbaren Fortsetzung von I a, ersichtlich ist, auf die stark verlangsamte, aber rhythmische Kammeraktion gar keine Wirkung; der Vagus schien gelähmt. Dass aber in Wirklichkeit keine Paralyse des Vagus besteht, sondern dass seine Wirkung nur durch die bestehende Dissoziation verdeckt ist, lässt sich in dem angeführten Beispiele leicht beweisen. Denn während der Vagusreizung ändert sich zwar nichts an der Schlagfolge der Kammern, wohl aber an jener der Vorhöfe, wie dies aus den nun weit auseinanderrückenden $P$-Zacken hervorgeht.

Es wurde nun der Effekt der Vagusreizung in kurzen Intervallen immer wieder geprüft. Nach kurzer Zeit, die Herzschläge waren inzwischen etwas frequenter geworden, erhielten wir bei einer gleichstarken Vagusreizung wie früher wieder deutliche Verlangsamung. Das jetzt aufgenommene Elektrokardiogramm welches in Fig. II reproduziert ist, zeigt, wie nach Aufhören der Vagusreizung die Frequenz wieder zuninmt, dass aber auch ausserhalh der Vagusreizung die vordem vorhandene Dissoziation vollständig aufgehört hat.

Nicht immer ist indessen die Dissoziation nachweisbar. Sehr häufig fehlt im Stadium der Unerregbarkeit der Vagi auch im Elektrokardiogramm jedes Zeichen von Vorhoftätigkeit. Aber ebensowenig wie es gestattet ist, eine absolute Ruhe der Vorhöfe zu erschliessen, wenn keine Kontraktionen derselben sichtbar sind, so ist es auch nicht richtig, aus dem Fehlen der Vorhofzacken, namentlich wenn nur eine Ableitung in Betracht gezogen wird, ohne weiteres auch eine vollständige elektrische Inaktivität der Vorböfe anzunehmen; deun wir wissen, dass unter diesen Umständen auch bei durchaus normalem Herzschlage mitunter bei einer bestimmten Ableitung keine Aktionsströme der Vorhöfe sich im Elektrokardiogramm nachweisen lassen. Da sich nun, wie gegenwärtig angenommen wird und wie es wobl auch für viele Fälle zutrifft, das Elektrokardiogramm bei Ventrikelautomatie :nur durch das Fehlen der P-Zacken von dem normalen Elektrokardiogramm unterscheidet, so war es nötig, die Selbständigkeit der Kammertätigkeit noch speziell nachzuweisen. 
Dies gelingt sehr leicht durch die von Hering ${ }^{1}$ ) angegebene Methode der Extrasystolen. Schaltet man nämlich in die Reihe der automatischen Kammerschläge eine Extrasystole ein, so ist dieselbe nicht mehr, wie bei der normalen Sukzession von Vorhofund Kammerkontraktionen, von einer vollständig kompensierenden Pause gefolgt, sondern die Extraperiode hat nunmehr annähernd die gleiche Dauer wie die spontanen automatischen Perioden. Auf diese Weise lässt sich denn auch bei vollständigem Fehlen der Vorhofzacken feststellen, dass die Ventrikel im Stadium der scheinbaren Unerregbarkeit der Vaği unabhängig von den Vorhöfen schlagen.

Fig. III zeigt neben dem Elektrokardiogramme auch die Suspensionskurven des rechten Vorhofes $(\boldsymbol{r} . A$.) und des rechten Ventrikels $(r$. V.). Diese wurden dadurch gewonnen, dass vor dem Belichtungsspalt des Aufnahmeapparates zwei an einem Ende in einer Klemme fixierte dünne Stahlfedern angebracht wurden, an deren freiem Ende mittels feiner Häkchen am rechten Vorhof und am rechten Ventrikel befestigte und über Rollen geleitete Fäden angriffen. Weder an der Suspensionskurve des Vorhofs, die nur yon der Kammertätigkeit herrührende Bewegungen zeigt, noch im Elektrokardiogramme ist die Vorbofstätigkeit nachweisbar. Die Form des Elektrokardiogramms weicht sonst von der normalen nicht $a b$; bei + wird durch mechanische Reizung des rechten Ventrikels eine Extrasystole ausgelöst. Die Dauer derselben ist nur um weniges länger als die der spontanen Perioden; die post-extrasystolische Pause ist weit davon entfernt, den gestörten Rhythmus zu kompensieren. Es handelt sich also um Kammmerautomatie, und tatsächlich war in diesem Stadium der Vagus auch bei R.-A. 0 chronotrop unerregbar.

In vielen, ja vielleicht in der Mehrzahl der Fälle von scheinbarer Vaguslähmung im Verlaufe der Muskarinvergiftung ist die Unabhängigkeit der Aktion der Ventrikel von jener der Vorhöfe auch bei Fehlen der $P$-Zacken unmittelbar aus der elektrographischen Kurve zu erkennen, indem dieselbe ausschliesslich solche Formen zeigt, welche Extrasystolen der recbten oder auch der linken Kammer entsprechen.

Ein Beispiel hierfür ist in Fig. IV abgebildet. Wir sehen hier

1) Hering, Nachweis der Automatie der mit den Vorhöfen oder Vorhofresten in Verbindung stehenden Kammern bzw. Verbindungsfasern des Säugetierherzens durch Auslösung ventrikulärer Extrasystolen. Pflüger's Arch. Bd. 107 S. 108.1905. 
in rhythmischer Folge den einzelnen Herzschlägen korrespondierende atypische Elektrokardiogramme von der charakteristischen Gestalt linksseitiger Extrasystolen: die Frequenz derselben wird durch eine der Marke entsprechende Vagusreizung (R.-A. 0) nicht im geringsten beeinflusst. Fig. IV zeigt überdies ebenso wie Fig. III die Suspensionskurven des rechten Ventrikels $(\boldsymbol{r}, \nabla$.) und des rechten Vorhofes (r. A.).

Erstere gibt ziemlich genau den Beginn der Kammerkontraktion wieder, ist aber sonst durch die der Kontraktion entgegenwirkende Federkraft entstellt und erscheint dadurch stark abgerundet. Die Suspeusionskurve des stillstehenden Vorhofes zeigt nur durch die Zusammenziehungen der Kammern veranlasste flache muldenförmige Senkungen und erst am Ende der Kurve einen einzelnen Schlag.

In Anbetracht des Umstandes, dass in dem durch Fig. IV repräsentierten Falle die Kammerkontraktionen linksseitigen Extrasystolen entsprechen, könnte die Frage aufgeworfen werden, ob wir es hier überhaupt mit einer echten Automatje der Kammern zu tun haben. Denn es wird bekanntlich gegenwärtig angenommen, dass die automatischen Kammerschläge im $\mathrm{H}$ is'schen Bündel, vielleicht im Tawara'schen Knoten, ihren Ursprung nehmen und deshalb, abgesehen von den. Fehlen der Vorhofzacke, ein normales Elektrokardiogramm liefern. Obwohl diese Frage für unsere Auffassung einer nur maskierten Erregbarkeit der Vagi in diesem Zustande des Herzens belanglos ist, erscheint es uns doch wichtig, hervorzuheben, dass eine solche scharfe Trennung von Kammerautomatie und extrasystolischer Ventrikeltätigkeit keine Berechtigung hätte. Es wäre denn, dass man in schärferer Weise als bisher nachweisen könnte, dass ein bestimmter Punkt des Leitungssystems - etwa der T aw ara'sche Knoten - sich durch eine ganz besondere Fähigkeit, rhythmische Reize zu produzieren, auszeichnen würde. Gegenwärtig ist es wohl am richtigsten, die rhythmische Aktion der aus ihrem physiologisehen Zusammenhange mit den Vorböfen in irgendeiner Weise getrennten Ventrikel kurz als Kammerantomatie zu bezeichnen.

In diesem Sinne können wir nun zusammenfassend sagen, dass die im Verlaufe der Muskarinvergiftung auftretende Unerregbarkeit der Vagi nicht einem Lähmungsustande derselben, sondern in allen Fällen dem Bestehenvon Kammerautomatie zuzuschreiben is t. 


\section{I1. Physostigmin.}

Ein anderes, in mancher Hinsicht vielleicht noch interessanteres Beispiel pseudotoxischer Vaguslähmung bietet das Physostigmin.

Der Hauptwirkung des Physostigmins, welche in einer mächtigen Steigerung der Erregbarkeit der Vagusendigungen besteht, steht noch immer der von $\mathrm{Harnack}$ und Witkowski am Froschberzen erhobene Befund unaufgeklärt gegenüber, dass nach erfolgter Vergiftung der Vagus gegen faradische Reizung unerregbar ist.

Diese beim Froschherzen in der Regel auftretende Reaktion haben wir in einzelnen Fällen auch bei Katzen und Hunden beobachtet. So sahen wir einmal gerade gelegentlich eines zur Demonstration der Erregbarkeitssteigerung bestimmten Versuches schon nach einer verhältnismässig kleinen Physostigmingabe $(1 \mathrm{mg})$ bei einer Katze absolute Unerregbarkeit der Vagi.

Wir fanden damals für dieses allen unseren Erfahrungen widersprechende Resultat keine andere Erklärung als höchstens die wenig befriedigende Annahme, dass in der Injektionsspritze Atropinreste von früheren Versuchen zurückgeblieben sein könnten. Gänzlich ratlos aber standen wir folgendem in Fig. V wiedergegebenen Versuche gegenüber.

Einem $8 \mathrm{~kg}$ schweren Hunde wurden $2,5 \mathrm{mg}$ Physostigmin intravenös injiziert, worauf zunächst, wie gewöhnlich, geringe Pulsverlangsamung und gesteigerte Erregbarkeit der Vagi eintrat. Später nahm die Pulsverlangsamung noch weiter zu; als wir jetzt den Vagus nochmals reizten (Fig. V 1. und 2. Marke) trat selbst bei R.-A. 0 keine Spur chronotropen Effektes ein. Wir hatten abermals jene paradoxe, beim Frosche allerdings fast regelmässig wahrzunehmende Reaktion vor uns. Von der übrigens unzutreffenden Vorstellung geleitet, dass es sich infolge der Physostigminapplikation um eine schon maximale, durch die hinzutretende faradische Reizung nicht mehr steigerungsfähige Vaguserregung handeln dürfte, injizierten wir, um letztere zu beseitigen, eine minimale Dosis Atropin ( $0,05 \mathrm{mg})$. Der Erfolg entsprach insofern unserer Erwartung, als darauf die Schlagfrequenz bedeutend zunahm und es sich bei neverlicher Prüfung der Vaguserregbarkeit (3. Marke) ergab, dass ein relativ schwacher faradischer Reiz (R.-A. $100 \mathrm{~mm}$ ) eine bedeutende und, wie Fig. V zeigt, fast 30 Sek. anhaltende Pulsverlangsamung erzeugte. Es war also nach Behandlung mit Atropin die dem Physostigmin eigentümliche Er- 
höhung der Erregbarkeit des intrakardialen Hemmungsapparates wieder zum Vorschein gekommen. Trotzdem mussten wir bald erkennen, dass die Voraussetzung einer maximalen Vaguserregung, von der wir ausgegangen waren, unzulänglich ist. In Übereinstimmung mit bereits in der Literatur vorliegenden Angaben fanden wir, dass auch nach lange fortgesetzter, die Frequenz stark herabsetzender Vagusreizung Verstärkung des Reizstromes oder Hinzufügung der Reizung des anderen Vagusstammes stets noch weitere Verlangsamung, ja selbst Herzstillstand hervorruft.

Erst unsere Erfahrung bei der Muskarinvergiftung und die analogen Ergehnisse der Untersuchungen bei der Physostigminintoxikation ermöglichten eine befriedigende Deutung dieses merkwürdigen Verhaltens.

Die Pulsverlangsamung war offenbar die Folge automatischer bzw. extrasystolischer Ventrikelkontraktionen. Diese wurden durch die antagonistische Wirkung des Atropins beseitigt, obne dass die angewendete kleine Dosis die Vagusfunktion wesentlich tangierte. Es konnte sogar jetzt die durch das Physostigmin gesteigerte Erregbarkeit des Vagus wenigstens teilweise noch hervortreten.

Dass es sich wirklich so verhält, haben wir durch spezielle Versuche nachgewiesen. Die scheinbare Lähmung des Vagus stellt sich bei der Physostigminvergiftung der Hunde allerdings nur in Ausnahmsfällen und gewöhnlich erst nach grösseren Dosen ein. Das Elektrokardiogramm zeigt aber dann in allen Fällen entweder ganz regellose, nicht weiter analysierbare Spitzen und Zacken oder die atypischen Formen von Extrasystolen.

Ein Beispiel hierfür geben wir in Fig. VI ${ }^{1}$ ). In diesem Falle war nach wiederholter Applikation von zusammen $9 \mathrm{mg}$ Physostigmin an Stelle der anfangs hochgradig gesteigerten Erregbarkeit des Vagus eine vollständige Wirkungslosigkeit desselben auch gegenüber der stärksten faradischen Reizung getreten. Das dargestellte Elektrokardiogramm lehrt nun in sehr anschaulicher Weise, dass der Grund des Versagens der Hemmungsnerven in der selbständigen Tätigkeit der Kammern gelegen ist.

Es ist daher in hohem Grade wahrscheinlich, dass die Eigenschaft des Physostigmins, automatische bzw. extrasystolische Ventrikel-

1) Diese Figur wurde durch Versehen der Kunstanstalt verkehrt abgedruckt. 
schläge auszulösen, auch die Ursache ist, dass beim Froschherzen im Zustande der Physostigminvergiftung Vagus- und Sinusreizung erfolglos bleibt oder nur eine verhältnismässig geringe Verlangsamung bedingt. Letztere beruht aber keineswegs auf chronotroper Hemmung der automatisch schlagenden Kammern, sondern vielmehr darauf, dass bei geringeren Graden der Vergiftung die automatische und daher langsamere Kammertätigkeit erst durch die Vagusreizung ausgelöst wird, wofür wir in Fig. VII ein in einem früheren Stadium der Vergiftung (1 mg Physostigmin) dem gleichen Versuche wie Fig. VI entnommenes Beispiel geben. Es handelt sich dabei um dieselben Vorgänge, welche unter Umständen auch zum Flimmern der Vorhöfe, ja selbst des ganzen Herzens, führen können. Dieselben bestehen darin, dass durch gewisse Gifte gesetzte, latente Reize durch den Einfluss des Vagus wirksam gemacht werden.

\section{Digitalin- nnd Digitaliskörper.}

Die schon seit Traube |bekannte vaguslähmende Wirkung der Digitaliskörper wurde erst vor wenigen Jahren von v. Lhota ${ }^{1}$ ) näher untersucht. Dieser Autor verfolgte die allmähliche Entwicklung der Vaguslähmung im Verlaufe der Digitalisvergiftung und fand so, dass hier ein anderer Mechanismus vorliegen müsse wie bei der Atropinlähmung der Vagi. Während bei vorsichtig abgestufter Atropinapplikation der Effekt der Vagusreizung gleichmässig immer schwächer wird (geringere Pulsverlangsamung und Blutdrucksenkung) und endlich ausbleibt, äussert sich nach v. Lhota die allmählich stattfindende Lähmung der Vagi durch Digitalisstoffe durch eine zunehmende Verlängerung der Latenzzeit, also durch einen immer mehr und mehr verzögerten Eintritt des im übrigen unveränderten Reizeffektes. Wird so die Latenzzeit unendlich gross, so kommt auch hier überhanpt keine Vaguswirkung mehr zustande.

Aus dem Intaktbleiben der Vagusreaktion bis zum Eintritte vollständiger Lähmung schliesst v. Lhota, dass dieselbe nicht, wie bei Atropin, durch eine Veränderung der Hemmungseinrichtungen, sondern durch eine Alteration der motorischen Funktionsfähigkeit des Herzens bedingt ist. Anhaltspunkte für diese Annahme findet v. Lhota

1) v. Lhota, Untersuchungen über die vaguslähmende Wirkung der Digitaliskörper. Arch. f. exper. Pathol. u. Pharmakol. Bd. 58 S. 350. 1908. 
darin, dass er feststellen konnte, dass die Dehnung der Latenz und die schliessliche Lähmung der Vagi durch Digitalis bei gleichzeitiger Einverleibung von Physostigmin, eines die Erregbarkeit des Herzmuskels steigernden Mittels, rascher erfolgt, und dass umgekehrt Apomorphin, „eine die Erregbarkeit des Herzmuskels herabsetzende Substanz", die sich bereits unter Digitaliseinfluss entwickelnde $\mathrm{Zu}$ nahme der Latenz wieder verkürzt.

Obwohl nun v. Lhota auf die Untersuchungen von Cushny ${ }^{1}$ ) und Brandenburg ${ }^{2}$ ) verweist, von denen namentlich der letztere nachgewiesen hat, dass durch mässig starke Gaben von Digitalin beim Frosche die Fähigkeit der Kammervorhofgrenze zur automatischen Reizerzeugung gesteigert wird, und dass bereits eine kurze nervöse Hemmung des Sinusgebietes durch reflektorische Vagusreizung genügte, um die Automatie in Erscheinung treten zu lassen, sagt er trotzdem mit einem gewissen Vorbehalt, dass die Unwirksamkeit der Vagusreizung bei Digitalisvergiftung auf Grund der erhöhten automatischen Erregbarkeit erklärt werden "könnte“. Und mit Rücksicht auf die rascher erfolgende Vaguslähmung bei kombinierter Digitalis-Physostigmin-Vergiftung interpretiert v. Lhota diese Erscheinung dahin, dass bei dem Zustandekommen der Vaguslähmung der Herzmuskel beteiligt sei, und zwar in der Weise, dass die erhöhte Erregbarkeit desselben die normale Vaguswirkung blockiert.

Mit dieser Auslegung schliesst sich aber v. Lhota, ähnlich wie z. B. Wybauw ${ }^{3}$ ), der die Bedeutung der Automatie bei der Vaguslähmung des mit $\mathrm{NaCl}$ oder mit anderen Flüssigkeiten gespeisten Schildkröten- und Froschherzens weitgehend berücksichtigte, im Grunde doch wieder der Meinung jener älteren Autoren an, welche die Wirkungslosigkeit der Vagusreizung nach Vergiftung mit Physostigmin, Kampfer, Digitalin (K $\operatorname{lug}^{4}$ ), Guanidin usw. darin erblickten,

1) Cushny, Journ. of exper. Medicine t. 2. 1897.

2) Brandenburg, Über die Eigenschaft des Digitalin, beim Froschherzen die selbständige Erzeugung von Bewegungsreizen an der Grenze von Vorböfen und Kammern anzuregen. Arch. f. Anat. u. Physiol. 1904 Suppl. S. 213.

3) Wybauw, Étude de certaines conditions dans lesquelles le nerf pneumogastrique cesse d'agir sur le cour. Arch. internat. de Physiol. t. 2 p. 198. 1904-1905.

4) Klug, Über die Wirkung des Digitalins auf die Blutgefässe und das Herz. Arch. f. Anat. u. Physiol. 1880 S. 457. 
dass die Hemmungsnerven gegen den direkt oder unter Vermittlung besonderer nervöser Einrichtungen (Klug l. c) zu stärkeren Kontraktionen gereizten Herzmuskel vergeblich ankämpfen.

Auch bei der Vaguslähmung durch Digitalis handelt es sich nicht um ein derartiges einfaches, mechanisches Gegenspiel von einanderentgegengesetzten Kräften, sondern vielmehr darum, dass die Erregung der Hemmungsnerven bei allen jenen Giften wirkungslos bleibt, welche den Herzmuskel von solchen Stellen aus zur Kontraktion anregen, diedemdirekten (chronotropen) Einflusse der Vagi von vornherein entzogen sind. Nicht die Stärke; sondern der Angriffspunkt gewisser toxischer Reize ist hier das entscheidende Moment.

Bei unseren Experimenten konnten wir bei Hunden häufig vollständige Unerregbarkeit der Vagi durch intravenöse Injektion von 2 bis $3 \mathrm{ccm}$ einer 1\% 1\%en Digitalinlösung im Verlaufe von 10-15 Minuten, erzeugen. Aus dem Elektrokardiogramm liess sich dann ohne Ausnahme feststellen, dass in diesem Stadium Ventrikelautomatie bestand.

In der weitaus überwiegenden Anzahl der Experimente zeigte cias Elektrokardiogramm die Formen linksseitiger und nur bei sehr vorgeschrittener Vergiftung manchmal auch die rechtsseitiger Extrasystolen. Wir müssen deshalb annehmen, dass die Digitalis ihren Angriffspunkt in den Kammern selbst hat, und dass der linke Ventrikel für die Giftwirkung besonders empfänglich ist.

Bei Anwendung kleinerer Digitalingaben $(1-1,5 \mathrm{ccm}$ der $1 \%$ igen Lösung) geht dem vollständigen Versagen der Vaguswirkung ein Zustand voraus, in welchem auch durch die stärksten Ströme nur eine Verlangsamung der Herztätigkeit erzielt werden kann. Fig. VIII a und $b$ (VIIIb ist die direkte Fortsetzung von VIII a), welche von einem Hunde stammt, der 15 Minuten früher 1,5 cem Digitalin ( $1 \%$ ) intravenös erhalten hatte, zeigt sehr deutlich den Mechanismus dieser Verlangsamung, der genau dem entspricht, welchen Brandenburg (l. c.) am Froschherzen beschrieben hat. Durch den Vagusreiz wird zunächst der Vorhof zum Stillstand gebracht. Die Dauer dieses Stillstandes und die den Reiz lange überdauernde Abschwächung der Vorhofkontraktionen ( $r$. A. Suspensionskurve des rechten Vorhofs) beweist, dass die Erregbarkeit der Vagi eher gesteigert ist. Trotzdem beginnen die Kammern nach einer ganz kurzen Pause wieder 
ziemlich rhythmisch zu schlagen. Die im linken Ventrikel offenbar bereits latent vorhandenen, durch den rascheren Vorhofrhythmus noch übertönten Impulse treten in dem Momente in Erscheinung, in welchem vom Vorhofe keine Reize mehr herabgelangen. Umgekehrt macht der ventrikuläre Rhythmus wieder dem Rhythmus des Vorhofes Platz, sobald die Vorhöfe zu ihrer ursprünglichen Schlagfrequenz zurückgekehrt sind (Fig. VIIIb).

Dass bei kombinierter Digitalin-Physostigmin - Vergiftung die Vagi ihren Einfluss auf die Kammern und damit auch auf Puls und Blutdruck früher verlieren, wie dies v. Lhota beobachtet hat, erklärt sich in sehr einfacber Weise dadurch, dass beide Gifte in ähnlicher Art selbständige Bewegungsreize der Kammern erzeugen. Diese toxischen Reize sind wenjgstens in dem in Fig. VIII dargestellten Stadium sicher schwächer als die normaler Leitungsreize, da sie anderenfalls auch ausserhalb der Vagusreizung zumindest einzelne Extrasystolen auslösen würden. Auch aus diesem Grunde haben wir keine Ursache anzunehmen, dass die Wirkung der Vagi durch die Stärke dieser abnormen Impulse blockiert wird.

Eine der Vaguslähmung vorangehende Verlängerung der Latenzzeit, wie sie v. Lhota bei Kaninchen und Katzen beobachtet hat, haben wir in unseren Versuchen an Hunden niçht gesehen. Der Grund für dieses differente Verhalten kann in der Art der Versuchstiere oder in der Dosierung gelegen sein; jedenfalls ist diese Dehnung der Latenzzeit keine konstante Erscheinung und darum für die Deutung der scheinbaren Vagusparalyse durch Digitaliskörper irrelevant.

Im übrigen ist uns diese Verlängerung der unter normalen Verhältnissen nur 1-2 Pulsschläge betragenden Latenzzeit der Vagusreizung nicht unbekannt; wir haben sie inshesondere bei der Vergiftung mit Calcium (unveröffentliche Versuche) und Physostigmin beobachtet, und der eine von uns [W interberg $\left.\left.{ }^{1}\right)\right]$ hat diese auffallende Tatsache bei der Physostigminvergiftung bereits früher beschrieben.

Diese Erscheinung beruht, wie wir uns nunmehr überzeugt haben, wenigstens bei der Physostigminvergiftung ebenfalls auf dem

1) Winterberg, Über die Wirkung des Physostigmins auf das Warmblüterherz. Zeitschr. f. exper. Pathol. u. Therap. Bd. 4. 
Auftreten automatischer linksseitiger Kammerkontraktionen im Beginne der Vagusreizung. Nach einiger Zeit können die letzteren sistieren, und der Vaguseffekt tritt in Erscheinung. Ja es kann sogar vorkommen, dass erst nach dem Sistieren der Vagusreizung diese automatischen Kontraktionen wegfallen, und dass dann erst - also in der Nachwirkung - vollständiger Herzstillstand eintritt. Das letztere merkwürdige Verhalten ist namentlich bei vorgeschrittener Calciumvergiftung nicht selten.

In den Versuchen von v. Lhota handelt es sich wahrseheinlich ebenfalls um solche automatische Kontraktionen im Beginne der Vagusreizung, die später sistieren. Dafür spricht der auch von v. Lhota hervorgehobene Umstand, dass während dieser „Latenzzeit" sich der Puls etwas verlangsamt und der Blutdruck sinkt. Überdies bemerkt man in den von dem Autor beigegebenen Pulskurven des ofteren arhythmische auf Extrasystolen zu beziehende Unregelmässigkeiten.

Schliesslich wollen wir noch hervorheben, dass kein einziger unserer Versuche einen Anbaltspunkt für einen chronotropen Einfluss des Vagus auf die automatisch schlagenden Kammern des mit Digitalin vergifteten Herzens gibt. Weder bei voll entwickelter Automatie noch in jenem Stadium, in welchem die Kammerautomatie erst durch die Vagusreizung provoziert wird, vermag Reizung der Hemmungsnerven den Kammerrhythmus zu verlangsamen. Da Hering und Rihl, zum Teil gerade auf Grund ihrer Experimente am digitalinvergifteten Herzen, für die Fähigkeit des Vagus, die automatisch schlagenden Kammern chronotrop zu hemmen, eintreten, sind unsere abweichenden Befunde immerhin von Interesse.

In manchen Fällen glaubten wir allerdings ebenfalls sowohl aus der Beobachtung des Herzens als auch aus dem aufgenommenen Elektrokardiogramm auf eine chronotrope Hemmungswirkung der Vagi auf die Kammern bei bestehender Dissoziation sehliessen zu müssen. Die nähere Kurvenanalyse ergab aber, dass es sich in allen diesen Fällen ausnahmslos um Überleitungsstörungen handelte.

Fin typisches Beispiel von Überleitungsstörung, welches freilich der Deutung keine Schwierigkeiten bereitet, geben wir in Fig. IX. Nach jedem vierten Vorhofschlage kommt es hier zum Ausfall einer Kammersystole, indem die Überleitungszeit ( $\mathrm{U}^{1}, \mathrm{U}^{2}, \mathrm{U}^{3}$ usw. gemessen durch die Distanz zwischen dem Beginne der Vorhof- 
kontraktion und dem Beginne der $R$-Zacke), nach jeder $A s$ länger wird, bis endlich die vierte $A s$ in die refraktäre Phase der Kammer füllt. Nach dem so entstandenen Kammersystolenausfall, wird die Überleitungszeit wieder erheblich kürzer, und der leicht zu durchschauende Vorgang beginnt von neuem.

Nicht so einfach ist die Überleitungsstörung in Fig. $X$ zu erkennen. Das Intervall $A s-R$ nimmt hier viel langsamer zu, bis nach $A s^{9}$ Kammersystolenausfall erfolgt. Insbesondere ist es in diesem Beispiele die Lage der $P$-Zacke, welche zu der fälschlichen Annahme einer bestehenden Dissoziation verleiten könnte. Wir sehen nämlich, dass die $P$-Zacke an einzelnen Stellen sich zwischen der $R$ und $T$-Zacke erbebt (nach $R_{5}$ und $R_{7}$ ), ja dass sie sogar in den katakroten Teil der $R$-Zacke (bei $R^{8}$ ) fallen kann. Diese ganz abnorme Situation der $P$-Zacke auf die wir besonders aufmerksam machen möchten, kommt dadurch zustande, dass infolge der zunehrnenden Überleitungszeit die nächste Aktion des Vorhofes schon zu einer Zeit beginnt, wo die vorangegangene verzögerte Kammersystole noch nicht abgelaufen ist.

Auch eine blosse Berücksichtigung des Zahlenverhältnisses $z$ wischen $A s$ und $V s$ bzw. $R$ könnte zu Irrtum Anlass geben, weil der $V s$-Ausfall nicht immer regelmässig nach derselben Anzahl von $A s$ erfolgt. So kommen in Fig. $\mathrm{X}$ auf $10 \mathrm{As} 8 \mathrm{R}$-Zacken; es besteht also scheinbar kein bestimmtes Verhältnis. zwischen Vorhofund Kammertätigkeit. Es handelt sich jedoch auch hier nur um Überleitungsstörung, indem einmal nach 4, das andere Mal schon nach 3 As Kammersystolenausfall eintritt.

Wenn überdies die durch den Ausfall eines Kammerschlages hervorgerufene Störung des Ventrikelrhythmus durch eine dem Systolenausfall vorangehende besonders bedeutende Verlängerung des Intervalls $A s-V s$ und eine dem $V s$-Ausfall folgende starke Verkürzung derselben zum grossen Teile ausgeglichen wird, können der Differentialdiagnose zwischen Dissoziation und Überleitungsstörung weitere erhebliche Schwierigkeiten erwachsen.

Ausser mit Digitalin haben wir auch mit Helleborein Versuche ausgeführt. Die Ergebnisse derselben, soweit sie sich auf das Versagen der Vaguswirkung beziehen, sind mit den eben geschilderten identisch. 


\section{Strychnin und Adrenalin.}

Auch im Verlaufe der Strychnin- und Adrenalinvergiftung kommt es häufig zur Vaguslämung. In allen Fällen, wo dieselbe eintritt, lässt sich bestehende, ebenfalls vorzugsweise linksseitige Kammerautomatie nachweisen. Umgekehrt ist stets chronotrope Vagushemmung erzielbar, solange das Elektrokardiogramm normal bleibt. In dieser Beziehung sind grosse Unterschiede vorhanden. Manchmal wurde nach Injektion von $1-2 \mathrm{mg}$ Strychnin der Vagus bereits unerregbar gefunden; in anderen Fällen bekamen wir selbst nach Dosen von 5-7 eg Strychnin immer noch Pulsverlangsamung. Der Effekt der Vagusreizung nach solchen kolossalen Dosen war allerdings vermindert; es kann also eine geringfügige, aber auch toxikologisch kaum in Betracht kommende, lähmende Wirkung grösster Strychnindosen auf den Vagus zugegeben werden.

Noch viel weniger konstant hinsichtlich der Erregung von Kammerautomatie wirkt das Adrenalin. Bei beiden Substanzen musste berücksichtigt werden, dass die Unerregbarkeit der Vagi auch von der Steigerung des intrakardialen Druckes abhängig sein könıte. Im folgenden Kapitel zeigen wir indessen, dass auch diese Form der Vaguslähmung nur auf Kammerautomatie beruht. Überdies lässt sich die Unabhängigkeit der Strychnin- und Adrenalinlähmung der Vagi von der Höhe des Blutdruckes dadurch nachweisen, dass sie noch bestehen bleiben kann, wenn der Blutdruck bereits zu seinem früheren Niveau zurückgekehrt ist.

\section{Steigerung des intrakardialen Druckes.}

Unerregbarkeit der Nn. vagi beobachtet man, wie gerade erwähnt, nicht nur infolge toxischer Einflüsse, sondern, wenngleich mit geringerer Regelmässigkeit, auch nach starken mechanischen Ein wirkungen.

So findet man in der Literatur mehrfach die Angabe, dass Vagusreizung keine chronotrope Hemmung mehr gibt, sobald das Herz unter hohem intrakardialen Druck steht ${ }^{1}$ ). Eine der alltesten hierhergehörigen Beobachtungen ist jene von Arnstein und Sustschinsky ${ }^{2}$, welche bei Klemmung des Aortenbogens auf der

1) Hofmann in Nagel's Handb. d. Physiol. Bd. 1 S. 264.

2) Arnstein und Sustschinsky, Über die Wirkung des Kalabar auf die Herznerven. Untersuchungen aus dem physiol. Laboratorium in Würzburg Bd. 2 S. 81. 1869. 
Höhe der Kalabarvergiftung mitunter eine vollständige Wirkungslosigkeit der Vagusreizung feststellen konnten. Sie erklären diese auffallende Erscheinung durch eine starke von der Drucksteigerung bewirkte Erregung des muskulo-motorischen Apparates, welche den Einfluss der Hemmungsnerven überkompensiert.

Später hat $\mathrm{Sustschinsky}^{1}$ ) in wenigstens zum Teile reineren, weil ohne Anwendung von Kalabar-Extrakt ausgeführten Experimenten den Einfluss des gesteigerten intrakardialen Druckes auf die Erregbarkeit des Vagus nochmals speziell untersucht und fand dieselbe sowohl bei Klemmung der Aorta als auch der Pulmonalis (Kaninchen) herabgesetzt oder vollständig aufgehoben. Er schliesst dennoch eine Paralyse der Hemmungsnerven aus und meint, dass bei der Zunahme des intrakardialen Druckes sowohl im rechten als im linken Herzen die Vaguserregbarkeit nur scheinbar vermindert, in Wirklichkeit aber bis zum Maximum erhöht wird und sich nur deshalb nicht äussern kann, weil sie die Widerstände in den stark erregten, antagonistischen motorischen Zentren nicht zu überwältigen vermag.

In ähnlicher Weise führt auch Tsehirjew ${ }^{2}$ ) (S. 156) ,jene sonderbaren Fälle", wo während der Blutdrucksteigerung Vagusreizung keine Verlangsamung bewirkt, auf den überwiegenden Einfluss des durch den erhöhten Druck gleichzeitig erregten Accelerans zurück; ds.s der schon von Baxt aufgestellten Regel widersprechende Überwiegen des Accelerans sucht Tschirjew durch die Annabme plausibel zu machen, dass der Hemmungsapparat in diesen Fällen schon von vornherein schwach entwickelt oder durch vorausgegangene Erregungen ermüdet sei.

Ebenso gibt Stewart3) an, dass die intrakardiale Druckerhöhung die Acceleranswirkung wahrscheinlich verstärkt, dagegen den hemmenden Einfluss des Vagus erst abschwächt und dann auf-

1) Sustschinsky, Zur Physiologie der peripherischen Endigungen des Vagus im Herzen. Untersuchungen aus dem physiol. Laboratorium in Würzburg Bd. 2 S. 159.1869.

2) Tschirjew, Über den Einfluss der Blutdruckschwankungen auf den Herzrhythmus. Arch. f. Anat. u. Physiol. 1877 S. 116.

3) Stewart, The influence of temperature and of endocardial pressure on the heart and particularly on the action of the vagus and cardiac sympathetic nerves. Journ. of Physiol. vol. 13 p. 59. 1892. 
hebt. Er nimmt deshalb an, dass die gedehnte Herzwand hemmenden Impulsen weniger zugänglich ist als augmentatorischen.

Auf eine gesteigerte Erregbarkeit des Vagus bei erhöhtem intrakardialem Drucke haben wiederum ältere Autoren zum Teil mit grossem Nachdrucke (Heidenhain ${ }^{1}$ ) die bei verstärktem Binnendruck auftretenden Unregelmässigkeiten zurückgeführt, weil dieselben auf der Pulskurve zugleich das Bild einer verlangsamten Herztätigkeit darboten.

Es ist das Verdienst von $\mathrm{Knoll}^{2}$ ), den Beweis erbracht $\mathrm{zu}$ haben, dass diesen auch nach Atropinisierung zustande kommenden unregelmässig verlangsamten Pulsen insuffiziente, abortive, oft sogar an Frequenz gesteigerte Herzschläge entsprechen, die durch direkte Einwirkung auf die Muskelfasern oder auf den motorischen Nervenapparat entstehen.

Herin $g^{3}$ ) erkannte endlich, dass diese Unregelmässigkeiten nichts anderes sind als durch die Druckerhöhung mechanisch ausgelöste Extrasystolen.

In dem Auftreten ventrikulärer Extrasystolen bzw. in der Erweckung ventrikulärer Automatie bei Sperrung oder starker Einengung der Abflusswege der rechten oder der linken Herzkammern liegt natürlich auch hier der Schlüssel für das Verständnis der relativen oder absoluten Wirkungslosigkeit der Vagusreizung bei Steigerung des intrakardialen Druckes, für den plötzlichen Wechsel von scheinbar vollständiger Vaguslähmung und normaler Hemmungswirkung und für die "kapriziöse" Weise, in welcher das Herz hinsichtlich der Funktion seines Hemmungsapparates so gut wie hinsichtlich des Auftretens von Arhythmie (Knoll l. c.) auf Steigerung des Binnendruckes reagiert.

Den Einfluss der Blutdrucksteigerung auf die Vaguserregbarkeit prüften wir gesondert sowohl am linken als auch am rechten Herzen durch temporäre Klemmung der Aorta bzw. der Pulmonalis.

1) Heidenhain, Über die arhythmische Herztätigkeit. Pflüger's Arch. 1872.

2) Knoll, Über die Veränderungen des Herzschlages bei reflektorischer Erregung des vasomotorischen Nervensystems usw. Sitzungsber. d. Akad. d. Wissensch. zu Wien Bd. 66 Abt. 3 S. 195.

3) Hering, Zur experimentellen Analyse der Unregelmässigkeiten des Herzschlages. Pflüger's Arch. Bd. 82 S. 1. 1900. 
In beiden Fällen konnte Herabsetzung der Vaguserregbarkeit im Sinne eines nur geringen oder gänzlich fehlenden negativ chronotropen Effektes erzielt werden; sowohl bei Widerstandserhöhungen für das rechte als auch für das linke Herz war jedoch die Inkonstanz auffallend, mit der das Versagen der Vaguswirkung auftrat.

Nach Sperrung der arteriellen Strombahn (mit Ausnahme einer zur Druckmessung dienenden Karotis) beobachteten wir selbst bei enormen Druckwerten von $360-380 \mathrm{~mm} \mathrm{Hg}$ mitunter noch vollständigen, mehrere Sekunden anhaltenden Herzstillstand, während noch kurz vorher bei demselben Versuchstiere sogar bei etwas niedrigeren Druckwerten keine chronotrope Hemmung wahrgenommen wurde. Diesem Verhalten begegneten wir besonders, weun schon wiederbolte Klemmungen vorgenommen worden waren.

Aus diesen Erfahrungen glauben wir schliessen zu dürfen, dass nicht der auf der Herzwand lastende Druck an und für sich, sondern die durch denselben und durch die gleichzeitige Behinderung der Blutentleerung bedingte mechanische Dehnung der Kammermuskulatur dem Versagen der chronotopen Hemmungswirkung zugrunde liegt.

Der scheinbar launenhafte Wechsel der Versuchsresultate, der zu vielfachen Kontroversen in der Literatur Anlass gab, dürfte von bleibenden und dann nicht mehr als Reiz wirkenden Dehnungen der Muskulatur sowie davon abhängig sein, ob und in welchem Grade im einzelnen Falle durch Klappeninsuffizienz die Überfüllung der Herzkammern ausgeglichen wird.

Wie in den früheren Fällen ist auch bei intrakardialer Drucksteigerung die scheinbare Vagusparalyse eine vollständige, wenn schon vor der Vagusreizung ventrikuläre Automatie eingetreten ist. Die Frequenz der selbständigen Ventrikelschläge kann dabei annähernd dieselbe sein wie jene der vom Vorbof abhängigen; ja sie kann sogar die letztere übertreffen. Denn die Regel, dass die automatischen Schläge langsamer sind als die normalen, gilt nur für den Fall, dass bei Wegfall der Vorhofimpulse in den Kammern keine abnormen Reize wirksam werden. Wenn aber, wie in den vorliegenden Experimenten besondere chemische oder mechanische Reize eingreifen, ist die Frequenz, mit welcher die Reaktion auf letztere erfolgt, neben dem Grade der Erregbarkeit des betreffenden Herzabschnittes in erster Linie von der Intensităt dieser Reize abhängig. 
Daher kommt es, dass die Schlagzahl bei erhöbtem intrakardialem Druck bald etwas verlangsamt, bald sogar beschleunigt ist. In dieser Weise finden die widersprechenden Befunde der einzelnen Autoren eine einfache Erklärung. Dadurch erledigt sich auch die von T'schirjew. (l. c.) gegebene unzulängliche Deutung, dass es sich dabei je nach der individuellen Entwicklung und Ermüdbarkeit um ein Überwiegen der hemmenden oder der motorischen Apparate handle, die beide in gleicher Weise durch die Steigerung des intrakardialen Druckes gereizt würden.

Tritt die Kammerautomatie erst während der Vagusreizung hervor, so zeigt die Pulskurve ebenfalls keinen Stillstand, sondern entsprechend der in diesem Zustande noch geringeren automatischen Reizerzeugung nur verlanqsante Schläge.

Von Wichtigkeit für unsere Auffassung ist endlich die Tatsache, dass das Elektrokardiogramm bei Klemmung der Aorta stets von links ausgehende, bei Sperrung der Pulmonalis immer rechts entspringende Extrasystolen anzeigt.

Der letztere Fall ist in Fig. XI abgebildet. Dieselbe wurde bei verschlossener Pulmonalis erhalten. Kräftige Vagusreizung (R.-A. $50 \mathrm{~mm}$ ), welche früher zu längerem Herzstillstand geführt hatte, ist nur noch auf den Vorhof wirksam. Die Ventrikelschläge zeigen das Elektrokardiogramm rechtseitiger Extrasystolen.

Schärfer und sicherer als jedes andere Verfahren verrät demnach das elektrographische den Zustand und den Ausgangspunkt heterotoper Reizbildung.

\section{Zusammenfassung.}

Aus den vorliegenden Untersuchungengeht hervor, dass die Unerregbarkeit der Vagi gegenüber faradischer Reizung bei Vergiftung mit Muskarin, Physostigmin, Digitalis, Strychnin, Adrenalin sowie bei hohem intrakardialen Drucke durch Kammerautomatie vorgetäuscht wird. Absolute chronotrope Unwirksamkeit der Vagi findet sich, wenn die Kammerautomatie schon vor der faradischen Reizung bestand; tritt dieselbe jedoch erst infolge der letzteren auf, so kommt es in der Regel zu einer geringen Verlangsamung (relative Unwirksamkeit). 
254 C. J. Rothberger u. H. Winterberg: Uber scheinb. Vaguslähmung etc.

\section{Figurenerklärung.}

Sämtliche Figuren sind von links nach rechts zu lesen. In allen elektrographischen Kurven entspricht $1 \mathrm{~mm}$ Ordinate $10^{-4}$ Volt; Zeitmarkierung in Fünftelsekunden. Die oberste Marke in den Fig. I b, II, IV, VI (verkehrt abgedruckt), VII, VIII a, VIII $b$ und XI entspricht einer faradischen Reizung des Halsvagus. Fig. $\nabla$ zeigt die Kurve des Blutdruckes aus der Karotis; Zeitmarkierung in Sekunden; die Marken entsprechen Vagusreizungen. Während der ungefähr in der Mitte der Fig. $V$ befindlichen Unterbrechung der Blutdruckkurve wurde Atropin intravenös injiziert. Die genauere. Beschreibung der einzelnen Figuren befindet sich im Texte. 